\title{
Sobre la pluralización (-n) de los adverbios en español: el caso de siempren
}

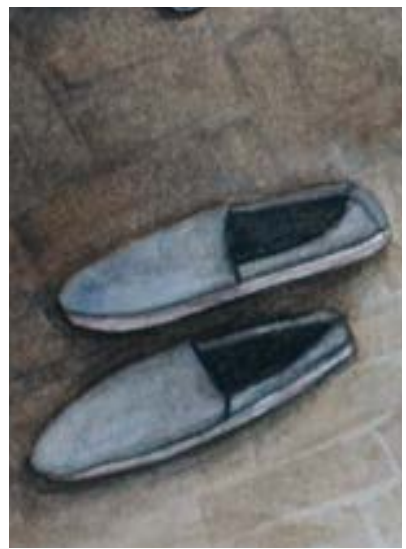

Enrique Pato

Universidad de Montreal, Canadá

enriquepato@hotmail.com

Trabajo recibido el $1^{\circ}$ de noviembre de 2019 y aprobado el 10 de agosto de 2020.

\section{Resumen}

El presente trabajo ${ }^{1}$ se centra en describir y examinar un caso concreto de pluralización adverbial (-n) en español actual (siempre $>$ siempren). Después de resumir los valores de la forma siempre, se presentan nuevos datos sobre este fenómeno de microvariación para conocer mejor su distribución geográfica en el mundo hispanohablante y los tiempos verbales con los que suele aparecer. A continuación, se ofrece una serie de consideraciones teóricas donde se muestra que el fenómeno se documenta en gallego y en catalán (sempren) y que esta concordancia puede ser entendida como un ejemplo más de la relación entre un controlador y un target, pero también que dos formas pueden recibir sus rasgos de una misma fuente en la estructura cognitiva, lo cual sería un proceso postsintáctico, y que este es uno más de los procesos de secuenciación y selección de elementos dentro del ordenamiento del discurso.

Palabras clave: español, variación, adverbio, pluralización, siempren

On the pluralization (-n) of adverbs in Spanish: the case of siempren

\footnotetext{
Abstract

This paper focuses on describing and examing a specific case of adverbial pluralization (in - $n$ ) in current Spanish (siempre > siempren). After summarizing the values of the form siempre ('always'), new data on this phenomenon

1 El presente trabajo ha sido financiado por el Conseil de recherches en sciences humaines du Canada dentro del proyecto No. CRSH-410-2018-0526. Mi agradecimiento a los revisores anónimos por sus comentarios y sugerencias de mejora, así como a los editores de la revista.
} 
of microvariation is presented to better understand its geographical distribution in the Spanish-speaking world and the tenses with which it usually appears. The following is a series of theoretical considerations that show that the phenomenon is also documented in Galician and Catalan (sempren), that this agreement can be understood as another example of the relationship between a controller and a target, but also that two forms can receive their traits from the same source in the cognitive structure, that it would be a postsyntactic process, and that this is one of the processes of sequencing and selection of elements within the discourse order.

Keywords: spanish, variation, adverb, pluralization, siempren

\section{Sobre a pluralização (-n) dos advérbios em espanhol: o caso de siempren}

\section{Resumo}

Este artigo se concentra em descrever e examinar um caso específico de pluralização adverbial (-n) no espanhol atual (siempre>siempren). Depois de resumir os valores da forma siempre, são apresentados novos dados sobre esse fenômeno da microvariação para melhor entender sua distribuição geográfica no mundo de língua espanhola e os tempos com que costuma aparecer. A seguir, é apresentada uma série de considerações teóricas que mostram que o fenômeno também está documentado em galego e catalão (sempren), que essa concordância pode ser entendida como outro exemplo da relação entre um controlador e um target, mas também que duas formas podem receber seus traços da mesma fonte na estrutura cognitiva, o que seria um processo pós-sintático, e que esse é um dos processos de sequenciamento e seleção de elementos dentro da ordem do discurso.

Palavras-chave: espanhol, variação, advérbio, pluralização, siempren

\section{Introducción}

La pluralización de los adverbios y cuantificadores es un aspecto de la gramática del español que solo ha empezado a ser tratada,de manera más o menos detallada, en trabajos recientes (Pato y Casanova 2017; Felíu Arquiola y Pato 2019; Pato 2019; Felíu Arquiola y Pato 2020). ${ }^{2}$ Los estudios llevados a cabo han mostrado que ciertos adverbios y cuantificadores pueden presentar formas flexionadas y varios patrones de concordancia. En efecto, dentro de la pluralización adverbial cabe distinguir dos procesos diferentes: i) la pluralización con -s (cercas, realmentes, cadas) y ii) la pluralización con -n (siempren).

El caso que nos ocupa en este trabajo (siempre > siempren) no aparece recogido en las obras académicas (RAE/ASALE 2005, 2009) ni en los

2 Hernández (2004, 109-110) documenta la pluralización de algunos adverbios en la prensa escrita canaria: fueron bastantes esperanzadores; van más despacios. Para un estudio detallado de estas y otras formas véase el trabajo de Felíu Arquiola y Pato (2020). 
trabajos generales sobre el español de América (Kany 1969; Fontanella de Weinberg 1993; Lipski 1996, entre muchos otros). Desde el punto de vista fonético, la adición de esa - $n$ podría interpretarse como un simple caso de paragoge (o adición de un sonido al final de una palabra). Sin embargo, como veremos en detalle, la aparición de la - $n$ está relacionada directamente con la forma verbal plural (V-n) que sigue al adverbio en el discurso, es decir, 'copiada', creando una relación de concordancia, tal y como comprobamos en los siguientes ejemplos (1a traen, 1b defienden, 1c intentan y 1d están), tomados de varios medios de comunicación en línea disponibles a través de Google y otros servidores. ${ }^{3}$

(1) a. Oblanca (PP-Foro) advierte de que los gobiernos socialistas siempren traen más paro para los asturianos (La Vanguardia.com, local, 20/04/2019, España).

b. Los padres que siempren defienden a los hijos en la empresa (Continental.com.ar, 16/12/2016, Argentina).

c. Manuel Pellegrini cree que "los jugadores siempren intentan engañar" (rpctv.com, deportes, 04/01/2014, Panamá).

d. Frustraciones, miedos, sorpresas y horrores siempren están a la orden del día en el mundo deportivo (Telemundo.com, deportes, 21/10/2019, Estados Unidos).

Según la propuesta teórica de Corbett (2006), cuanto más pequeño es el dominio en el que se da la concordancia, más canónica es esta. En otras palabras, la concordancia es más regular y conforme cuando el elemento controlador de la concordancia y el target (u objetivo) pertenecen al mismo sintagma. En nuestro ejemplo, el controlador es el verbo que irradia los rasgos de número, desde un dominio pequeño o dominio local que es el SV en el que aparece el adverbio siempren (como target), cuyo núcleo es el verbo controlador de la concordancia (V-n).

Por otro lado, cabe recordar que la jerarquía de implicación de Moravcsik (1974), establecida en términos de funciones gramaticales, clasifica la concordancia adverbial como última opción en las lenguas naturales: sujeto > objeto $>$ objeto indirecto $>$ adverbio.

Cabe destacar el carácter interdisciplinario del fenómeno en estudio. En efecto, desde la psicolingüística ejemplos como el de siempren han sido tradicionalmente objeto de estudio y se han explicado como lapsus linguae; en concreto, como un tipo de error por desplazamiento (del Viso 2002, 366). Desde el ámbito de la producción del lenguaje estos errores espontáneos, cometidos de manera involuntaria, no son considerados producto de ningún estado mental (afasia, problemas neuronales) ni dependen del nivel cultural de los hablantes. Además, no son arbitrarios, sino que se incluyen dentro de un fenómeno sistemático más general sometido a reglas (del Viso 2002, 356). Los lapsus linguae constan de dos elementos: el elemento anómalo (error) y el elemento pretendido (target). En algunas categorías podemos encontrar también el elemento origen (o fuente del error).

3 En los ejemplos que ofrezco a lo largo del trabajo, indico la fuente principal, el autor, el título de la obra literaria, la fecha de publicación y el país del medio de prensa, autor o usuario. En los casos del Corpus del español: Web/ Dialectos figura solo el país. Ninguno de los ejemplos ha sido editado o corregido, mantengo la escritura original con sus faltas ortográficas, algunas de ellas señaladas mediante [sic]. 
La clasificación tradicional de los lapsus linguae, siguiendo a Lecours y Lhermitte (1969), incluye básicamente dos categorías: i) rasgos que aparecen en un lugar donde no deberían aparecer, y ii) rasgos que faltan en el lugar donde deberían estar presentes. Siguiendo de nuevo a del Viso (2002), esta clasificación incluye los errores de movimiento o contextuales (movimiento de A a B) y los errores no contextuales. ${ }^{4} \mathrm{El}$ rasgo que interesa parcialmente para los fines de este trabajo es el de desplazamiento, es decir, los casos en que un elemento se desplaza (Ellos siempren viene, por Ellos siempre vienen). En el ejemplo anterior, "los elementos afectados, además de dejar vacía la posición que les correspondía [...] van a parar a una posición igualmente vacía, es decir, no sustituyen a ningún otro elemento, sino que simplemente se añaden" (del Viso 2002, 366-367). Volveremos sobre todo ello en $\S 4$.

Los objetivos de este trabajo, de carácter descriptivo y exploratorio, son varios. Por un lado, interesa conocer un poco mejor los posibles patrones de concordancia adverbio-verbo en español. Por otro, describir la aparición de un lexema (- $n$ ) que no pertenece a la categoría del adverbio y caracterizar la pluralización que sufre el adverbio siempre en español actual,gracias a los datos de varios corpus lingüísticos.

En este sentido, el trabajo queda estructurado de la siguiente manera. Tras esta introducción ( $§ 1$ ), me centro en presentar — de manera breve- los valores de siempre en español moderno (§ 2), para después ofrecer nuevos datos sobre su pluralización (siempren), conocer su distribución geográfica y los tiempos verbales con los que suele aparecer (§ 3). En el siguiente apartado (§ 4), presento y reviso una serie de consideraciones teóricas, en sentido interdisciplinar, sobre la pluralización adverbial desde distintas áreas de estudio. Las conclusiones, por último, cierran el trabajo (§ 5).

\section{Valores de siempre}

Los significados básicos del adverbio y cuantificador universal siempre que ofrece el Diccionario de la lengua española (RAE/ASALE 2017) son dos: i) 'en todo tiempo o en cualquier tiempo', y ii) 'en todo caso o cuando menos'. Por su valor, es una forma que convierte la predicación en habitual 'en todas las ocasiones' (Siempre va al cine con un amigo) (RAE/ASALE 2009, 1143). Sin embargo, no es cuantificador cuando se emplea con el valor de 'definitivamente, sin duda alguna' (Siempre no salgo mañana de viaje), uso propio de las normas de México, Centroamérica, el Caribe y los Andes (RAE/ASALE 2009, 1391). También puede crear una locución

\footnotetext{
4 Entre los errores de movimiento cabe incluir: i) la copia: cuando el elemento origen y el error son iguales, esto es se copia el primero en un lugar no correcto, ya sea por anticipación (te fongas (pongas) la falda) o por perseveración (el fiscal está a funto (punto)); y ii) el intercambio o metátesis (jamor de Yon (jamón de York)). Por su parte, los no contextuales se dividen en: i) sustitución: cuando el elemento target es sustituido por otro elemento ajeno (son concursos (recursos) limitados); ii) fusión: cuando el reemplazo de elementos es solo parcial y el resultado es un híbrido (ya hemos roto el fuego (roto el hielo + abierto el fuego); iii) omisión: cuando hay una ausencia y los elementos no aparecen en el lugar indicado (las topas (tropas) croatas); y iv) adición (son de claustura (clausura)) (cf. del Viso 2002).
} 
temporal-condicional, siempre que, con un significado próximo a 'todas las veces que, en todas las ocasiones que' (RAE/ASALE 2009, 1958).

Por otro lado, hay que indicar que este adverbio presenta otros significados contextuales posibles en las variedades americanas, y así aparecen recogidos en el Diccionario de americanismos (ASALE 2010). En concreto, cinco son las acepciones que ofrece este diccionario, a saber:

(2) a. 'Por fin, finalmente' (México, El Salvador, Nicaragua, Costa Rica, Panamá, Colombia, Perú, Bolivia).

b. 'Decididamente' (Cuba, Colombia, Bolivia).

c. 'A pesar de todo' (Chile, Perú, Bolivia).

d. 'Todavía' (El Salvador, Costa Rica, Perú, Bolivia).

e. 'De todos modos' (Perú).

Retomaré estas acepciones, más adelante, en la descripción de la forma en estudio.

\section{Nuevos datos}

Aunque en este trabajo me detengo en la situación del español actual, es importante señalar que la documentación de la forma siempren es histórica. ${ }^{5}$ En efecto, ejemplos de este adverbio pluralizado-concordado se pueden registrar en el siglo XVIII (3a), en el XIX (3b-c) y en el XX (3d). ${ }^{6}$

(3) a. Estas personas pues que siempren están asustadas, desconfiadas, y que tienen sentimientos indignos de su bondad, le hacen una grande injuria (Marqués de la Regalía, La esperanza christiana, 1794, España).

b. Siempren confunden los señores deístas la libertad del pensamiento con la de la lengua, por eso viven engañados y caen en la infamia de rebeldes (José de San Bartolomé, El duelo de la Inquisición, 1814, España).

c. “es indispensable que nuestras quejas lleguen á los oidos del rey, pues no es justo que mientras nos meten gato por liebre, adulterando la moneda, deje Su Alteza consumir espuertas de oro á esos judíos y moros que siempren lo rodean... (Nicasio Camilo Jover, Las amarguras de un rey, 1856, España).

d. Siempren encuentran los picaros quién los defienda, y los jueces los dejan escapar las más de las veces, con el pretexto de que no hay pruebas (Alfonso M. Maldonado, Cuentos y narraciones, 1908, España).

Los datos que empleo para la descripción de este uso proceden de diversas fuentes: el CORPES XXI (RAE), la red social Twitter y, sobre todo, el Corpus del español: Web/ Dialectos (Davies 2016). ${ }^{7}$

5 Evidentemente, quedan por fuera los casos de amalgama entre el adverbio siempre y la preposición en, como en el siguiente ejemplo: Pero por dios consagrado/ ques verdad lo que dire/ que peno mas ausentodo/ y quesiemprenmi he hallado/ quanto mas lexos mas fe (Pedro Barrantes Maldonado, Romances, c. 1534).

6 Solo el ejemplo (3c) procede del CORDE (RAE), el resto son fruto de las búsquedas del autor.

7 La forma siempren se documenta también en las variedades del español en la diáspora, y así aparece en los datos del Corpus oral de la lengua española en Montreal (Pato 2014 -2020): Por suerte, con mi familia, de parte de mi papá, que está aquí en Canadá, siempren organizan fiestas y... ahí suelo bailar (COLEM-Ecuador, M, 23 años). 
En el CORPES XXI se registran tres ejemplos de siempren en la prensa escrita, especialmente en entrevistas, en Venezuela (4a), Argentina (4b) y Panamá (4c). Estos datos son interesantes, ya que muestran el empleo de esta forma pluralizada, pero no permiten llevar a cabo una descripción detalladadel fenómeno en estudio.

(4) a. "Tuve algunos problemas debido a lo fuerte del terreno. A los tres kilómetros bajé el ritmo debido a un intenso dolor en el abdomen”, explicó Richard Carvajal. Más adelante agregó que "realizó una gran carrera, porque los kenianos siempren son rivales muy difíciles de superar" (Últimas Noticias.com.ve, 25/05/2001, Venezuela). b. "Sinceramente, para mí las cabañas están todas en primera línea y puede ganar cualquiera; no es como eran años atrás que siempren ganaban las mismas. Eso demuestra el avance de la raza, en donde nadie tiene el premio asegurado", agregó (La Nación.com.ar, 24/07/2004, Argentina).

c. “Nuestros fans siempren están ahí ayudándonos. Esta vez será especialmente duro y necesitamos que nos den una mano. Nosotros lo daremos todo, pero ellos deben llevarnos aún más lejos”, dijo Gennaro Gattuso, que cree que el ambiente en San Siro será crucial (Prensa.com, 02/05/2007, Panamá).

Para completar los objetivos previstos en este trabajo utilizo, por tanto, el Corpus del español: Web/ Dialectos, ya que los 129 casos registrados en este corpus permiten establecer una descripción algo más completa del uso de siempren en el mundo hispanohablante.

En concreto, los ejemplos documentados de siempren delante de un verbo en tercera persona de plural (V-n) llegan a 120 casos $(93,02 \%$ del total de la muestra), frente a 4 casos $(3,10 \%)$ en que siempren aparece después del verbo en tercera persona de plural. También se documentan 2 casos (1.55\%) de SN plural + siempren y otros 3 de locuciones: como siempren ( 2 casos, $1.55 \%$ ) y así siempren (1 caso, $0,77 \%$ ). El hecho de que siempren manifieste concordancia plural con el verbo finito viene sugerido por los datos del corpus manejado, ya que se presenta en la gran mayoría de las ocasiones con verbos plurales $(97,67 \%, 126$ casos de 129). No obstante, también es posible documentar la forma siempren con verbos singulares, aunque este patrón es muchísimo menos frecuente $(2,33 \%, 3$ casos de 129), y quizá sean erratas. El exponente $-n$ es, por tanto, un exponente de tercera persona plural. ${ }^{8}$

Con todo, la primera característica que se puede señalar en el uso de la forma siempren es que este adverbio aparece de manera categórica delante del verbo (V-n), en posición interna. ${ }^{9}$ Por otro lado, siguiendo a Corbett (2006), y como se vio en la introducción, estaríamos ante un caso de concordancia canónica y de atracción del plural. ${ }^{10}$

8 Los casos de primera persona plural (V-mos) con siempren son escasísimos (cf. 8d), al igual que sucede con otros adverbios pluralizados: todavían estamos corrompidos de parásitos politicos (Guatemala). Volveré sobre esta última forma en $\S 4$.

9 En el caso de siempre, y según los datos del Corpus del español: Web/ Dialectos, esta forma aparece delante del verbo en mayor proporción (67,55\%, 629.879 casos) que pospuesta al verbo (32,45\%, 302.620 casos).

10 Entiendo la atracción como "the cognitive processes involved in implementing agreement during language production” (Bock et al. 2001, 85). En este sentido, la atracción es más fuerte entre elementos de la misma cláusula y con formas en plural. 
La siguiente tabla muestra el número de casos, el porcentaje sobre el total (129 casos) y su frecuencia normalizada, es decir, el número de ocurrencias teniendo en cuenta el número de palabras de cada país en el corpus revisado.

\begin{tabular}{|c|c|c|}
\hline País & Casos (\%) & Frec. norm. \\
\hline Argentina & $8(6,20 \%)$ & 0,043 \\
\hline Bolivia & $2(1,55 \%)$ & 0,046 \\
\hline Chile & $1(0,77 \%)$ & 0,014 \\
\hline Colombia & $13(10,07 \%)$ & 0,072 \\
\hline Costa Rica & $3(2,32 \%)$ & 0,094 \\
\hline Cuba & $4(3,10 \%)$ & 0,059 \\
\hline Ecuador & $5(3,87 \%)$ & 0,087 \\
\hline El Salvador & $1(0,77 \%)$ & 0,025 \\
\hline España & $30(23,25 \%)$ & 0,065 \\
\hline Estados Unidos & $10(7,75 \%)$ & 0,055 \\
\hline Guatemala & $4(3,10 \%)$ & 0,066 \\
\hline Honduras & $2(1,55 \%)$ & 0,051 \\
\hline México & $18(13,95 \%)$ & 0,072 \\
\hline Nicaragua & $1(0,77 \%)$ & 0,028 \\
\hline Panamá & $1(0,77 \%)$ & 0,040 \\
\hline Paraguay & o (०\%) & 0,000 \\
\hline Perú & $15(11,62 \%)$ & 0,130 \\
\hline Puerto Rico & $1(0,77 \%)$ & 0,028 \\
\hline República Dominicana & o (०\%) & 0,000 \\
\hline Uruguay & $1(0,77 \%)$ & 0,024 \\
\hline Venezuela & $9(6,97 \%)$ & 0,083 \\
\hline Totales & $129(100 \%)$ & -- \\
\hline
\end{tabular}

Tabla 1: Número de casos, porcentajes y frecuencia normalizada de siempren.

Como observamos en la tabla precedente, todos los países hispanohablantes, salvo Paraguay y República Dominicana, aparecen representados en los datos del corpus para el uso de siempren. Esto no significa que en estos dos países mencionados no haya casos de pluralización de siempre, sino simplemente que en este corpus no se han documentado. ${ }^{11}$ Por otro lado, entre los países que obtienen mayor cantidad de ejemplos están España (30 casos, 23,25\%, cf. 5a), México (18, 13,95\%, cf. 5b), Perú (15, 11,62\% cf. 5c) y Colombia (13, 10,07\%, cf. 5d, donde vemos un caso de alternancia siempren/

11 De hecho, en la red social Twitter es posible registrar casos de siempren en estos países: Los grandes siempren vuelven (Twitter, nico_carballo16, 21/og/2016, Paraguay); Las Mujeres Siempren Necesitan un closet nuevo lleno de ropa nueva (Twitter, Yorbi_DeLeon, 04/o9/2014, República Dominicana). Por tanto, siempren se registra en todas las variedades del español actual, aunque con diferente frecuencia. 
siempre). Sin embargo, la frecuencia normalizada nos indica que es Perú (con 0,130$)$ el país con mayor índice de empleo de la forma siempren.

(5) a. La distribución tradicional de roles suponía que los hombres buscaran dominar su relación, mientras que las mujeres siempren tenían un sí, cariño en la boca para evitar discusiones (España).

b. Cuando he tratado de llegar a algo con muchas mujeres hay unas que siempren me ponen un obstáculo, me ponen el camino duro y difícil (México).

c. Pero la verdad que los juegos que siempren llaman mi atención son las versiones portátiles (Perú).

d. Aprendan que las clases pudientes siempren gobernarán a Colombia y siempre excluirán a los pobres... Despierten (Colombia).

La segunda característica en la descripción de esta forma adverbial pluralizada es que se trata de un uso más o menos generalizado en español actual. No obstante, Perú es el país donde mayor empleo se hace de siempren. A este respecto hay que recordar que Perú es uno de los dos países donde siempre puede presentar más significados (cf. 2).

En cuanto al tiempo verbal con el que aparece este adverbio, los datos del corpus indican que el más empleado es el presente de indicativo (96 casos de los 129 registrados, 74,41\%), como ejemplifico en (6). Le siguen el futuro de indicativo (13 casos, 10,07\%, siempren gobernarán) y el pretérito perfecto compuesto (10 casos, 7,75\%, siempren han hecho eso). En menor proporción, el pretérito imperfecto de indicativo (6 casos, 4,65\%, siempren se quejaban), el presente de subjuntivo ( 3 casos, 2,32\%, que siempren la recuerden) y el pretérito perfecto de subjuntivo (1 caso, $0,77 \%$ ).

(6) a. Lo que quiero mostrar son esos websites que siempren logran desconcentrarme y desviarme de mi trabajo (Costa Rica).

b. Los grandes hombres de la historia siempren dejan un legado para la humanidad (Venezuela).

c. sé firme en tus pensamientos, conserva la terquedad de un niño, ellos siempren luchan por conseguir lo que quieren (Ecuador).

d. Estos tipos de dictadores siempren buscan un enemigo externo para echarles la culpa de todo lo que pasa (Estados Unidos).

Además, el adverbio siempren puede aparecer precedido de la negación no, aunque el número de ejemplos no es muy abundante en el corpus manejado (8 casos de los 129 registrados, 6,20\%). En cambio, no se documentan ejemplos de la locución adverbial enfática siempren no ('decididamente no') ni de siempren sí ('decididamente sí'), cuya forma estándar ( pluralización) es propia de México, Nicaragua y Costa Rica (ASALE 2010).

(7) a. Capichi!!!! se nota que las neuronas no siempren responden. Igual es mi humilde opinión (Argentina).

b. El problema es que los pensamientos no siempren son los mismos, dependiendo de las circunstancias (Colombia).

c. Los músicos más exitosos no siempren son los más talentosos. Son aquellos que se mantienen implacables (México). 
d. GearBox presenta sus juguetes con gran alboroto, no siempren llegan a calar hondo como paso con su Duke Nukem (España).

La tercera característica, por tanto, es que siempren depende de un verbo en tercera persona de plural (V-n) en presente de indicativo $(74,41 \%),{ }^{12} \mathrm{y}$ - como cabría esperar- admite la negación antepuesta sin problema. Sin embargo, la distribución con respecto a la negación muestra que no es una propiedad que defina a siempren, sino que lo asimila a siempre.

Por lo que respecta a los valores de siempre que vimos en (2) para las variedades americanas, hay que indicar que no se documentan en el caso de siempren. En efecto, los datos revisados señalan que cuando el adverbio siempre se pluraliza mantiene su significado base ('en todo tiempo/ circunstancia, 'en toda ocasión/al menos'). En los ejemplos del corpus siempren no presenta el significado de 'por fin, finalmente' (como en ¿Fueron siempre al cine?), 'decididamente' (Deben apoyar siempre los procesos de paz) ni 'todavía' (¿Estarán siempre los jugadores veteranos?). Una posible razón de este hecho es que el adverbio siempre suele aparecer en estos casos detrás del verbo (V-n), no delante, contexto que favorece la pluralización y concordancia. Tampoco hay registros con las acepciones populares de 'a pesar de todo' y 'de todos modos' (ASALE 2010).

La descripción que he presentado hasta ahora de la forma siempren se puede completar con otros casos registrados en otro tipo de fuentes más coloquiales, como la red social Twitter. En efecto, los datos de Twitter nos muestran que la pluralización de siempre también se produce en otros contextos, a saber: cuando sigue a un sujeto plural y precede a un adjetivo plural (cf. 8a, Brays y Miriam... juntos), como adverbio en posición final tras un imperativo (cf. 8b), y como adverbio ante un verbo en singular, pero con un complemento SN plural (cf. 8c, tus comments). Por último, es posible documentar esporádicamente el uso de siempren con el verbo en primera persona de plural y un complemento en plural (cf. 8d, Cometemos... los mismos errores, y lo he señalado en la nota 8$).{ }^{13}$ Por tanto, en la mayoría de estos casos hay una forma plural (que subrayo en cada ejemplo) que serviría de elemento controlador de la pluralización del adverbio.

(8) a. Brays y Miriam siempren juntos (Twitter, carlofdez, 25/01/2018, Gijón, España). b. A manenti dámelo siempren (Twitter, SachaQuinteros, 16/10/2019, Santiago del Estero, Argentina).

c. Hola Jenny que bueno verte por aqui, siempren leo tus comments (Twitter, JzdeMiami, 12/10/2019, Miami, Estados Unidos).

d. Cometemos siempren los mismos errores solo para darnos cuenta de lo pendejos que somos (Twitter, AxelRous_, 17/09/2014, México).

12 En el caso de siempre, y según los datos del Corpus del español: Web/ Dialectos, el presente de indicativo también es el tiempo verbal que más se emplea con este adverbio ( $53,24 \%, 496.541$ casos).

13 Hecho que también se registra con los clíticos en las formas verbales (digamoslon) (cf. Mare 2018). 


\section{Consideraciones teóricas}

Después de ofrecer una descripción detallada de la forma siempren, varias son las consideraciones teóricas que voy a presentar ahora con ánimo de aportar luz nueva sobre este fenómeno morfosintáctico de microvariación. Por la naturaleza misma de este trabajo, estas consideraciones son interdisciplinares y preliminares.

En primer lugar, desde el punto de vista tipológico (microrregional), es interesante comprobar que el mismo fenómeno de pluralización se registra en otras lenguas romances peninsulares como el gallego (cf. 9, sempren) y el catalán (cf. 10, sempren), hecho que comprobamos en los siguientes ejemplos de la prensa escrita y blogs en línea.

(9) a. Víctor Sixto valorou o "asociacionismo maduro" do comercio galego. "Sempren falan dun comercio en positivo, que trata de colaborar cos concellos e coas institucións. Non buscan a confrontación", di (El Correo gallego.es, 03/11/2008).

b. Somos os bravos do norte, os que sempren tiran para diante por moito que nos boten. Lume nos montes, caciquismo nas vilas ou merda nas costas (Galicia Hoxe. com, 08/08/2009).

c. Manuel Torrado dijo al respecto que "os políticos veñen e marchan pero sempre están os técnicos, os que sempren levan a cabo as actividades. Son eles os grandes responsables de que todo saia ben" (La Voz de Galicia.es, 21/06/2013).

d. Houbo tamen filósofas, pese ás dificultades de formación, pero ningunha trascendeu de igual maneira que os seus colegas varóns. Elas sempren aparecen vinculadas a algunha figura masculina: pais, maridos, amantes, profesores (Diario de Pontevedra. es, 21/10/2019).

(10) a. els falangistes sempren han sigut republicans como bons feixistes (Racó Català.ca, 17/08/2010).

b. No a les dictadures que sempren sagnen els pobles i no als sàtrapes, siguin extravagants o no (e-Notícies.cat, 17/04/2011).

c. Disposo de carnet de conduir i de vehicle. No tinc fills pero els nens sempren han estat presents a la meva vida ja sigui... (Top Nanny.es, 20/10/2019).

d. Venga Tomeu!!!! (Tom) qu'estás emprenyat per culpa d'es Joker (Jerry)??? Tan mateix amb aquest "assunto" sempren serem en Tom y Jerry (Última Hora.es, 24/07/2014).

Estos casos de pluralización de la forma sempre siguen el mismo patrón que en español, ya que aparecen mayoritariamente con formas verbales de tercera persona de plural (V-n). En mucha menor proporción, sempren también se presenta con formas verbales en primera persona de plural (10d, serem, cf. el ejemplo de 8d para el español).

En segundo lugar, como vimos en la introducción, tipológicamente se ha entendido que los rasgos de concordancia se copian de los controladores formales a los targets (Corbett 2006). Sin embargo, otros autores conciben la concordancia de manera diferente a la relación controlador-target y sostienen que las formas lingüísticas coinciden entre sí porque reciben sus rasgos de una misma fuente en la estructura cognitiva. 
Según Kibrik (2019), la concordancia reside en los procesos discursivos cognitivamente motivados y asociados con la referencia. Así las cosas, la concordancia no sería un mecanismo gramatical autocontenido, sino un efecto secundario del 'mapeo' general cognición-forma (Kibrik 2019, 50). En este sentido, la concordancia es entendida como un fenómeno basado en el discurso y en el uso, en el que "two or more forms agree with each other in certain features because each of these forms relates to a cognitive entity bearing these features" (Kibrik 2019, 39).Como indica uno de los evaluadores, para el caso de siempre esta postura parece ser problemática, ya que siempren no tiene un referente obvio (como cuantificador). No obstante, si se postula que el exponente - $n$ está asociado a la cuantificación universal, el análisis se podría extender fácilmente a otras formas, como veremos más adelante. ${ }^{14}$

Con todo, sería el control nocional-conceptual (de [pluralidad] en este caso) el que actuaría sobre los rasgos de concordancia. Por ello, cuando dos formas están de acuerdo con un controlador mental, el resultado es una concordancia en paralelo, es decir, la concordancia se ve influenciada sintácticamente por la proximidad extrema de las formas (siempren $+V-n)$. En otras palabras, la estricta adyacencia o contigüidad produce que el adverbio siempren incida sobre el verbo (V-n), se delimiten semánticamente, y juntos formen una unidad superior. Esto es lo que hemos visto en los ejemplos precedentes del español (y del gallego y catalán).

En tercer lugar, desde el marco formal es sabido que los mecanismos gramaticales como Movimiento y Fusión, así como el orden de las proyecciones funcionales en sintaxis (Cinque 1999), bloquean los procedimientos de verificación de características (o chequeo de rasgos) para estas proyecciones, a menos que el proceso sea de 'último recurso'. En concreto, me refiero al Principio de fusión tardía (Late Merge Principle ${ }^{15}$ y a la Estrategia de último recurso (Last Resort Strategy). Siguiendo a Chomsky $(1995,136)$, los elementos léxicos no marcados con theta (no relevantes para la estructura theta) pueden esperar para fusionarse fuera del SV, ${ }^{16}$ esto es, pueden unirse a AGR (concordancia) para verificar la persona y el número. ${ }^{17}$ En el caso de siempren, esta concordancia del adverbio con el verbo (V-n) podría entenderse de la misma manera — para el número $(-n)$ - , al ser una concordancia de un rasgo-phi interpretable, que envuelve una representación semántica, pero que tiene lugar en un nivel postsintáctico, es decir, entre la sintaxis y la fonología. En concreto, siguiendo a Ackema y Neeleman (2004), en la Forma Fonética se expande y adquiere el número plural (proceso de copia de los valores de V) y se identifican los rasgos por adyacencia lineal

14 Como es sabido, en español se documentan otras formas "plurales" en - $n$, como nuncan o nadien. Sin embargo, el caso de nadien no parece recibir su $-n$ a partir de concordancia plural en todos los ejemplos (compárese Nadien dijo eso con Nadien nos van a entender).

15 Según el Late Merge Principle (LMP), la inserción léxica (external merge) es preferible in situ en lugar de requerir movimiento o copiado (internal merge).

16 En este sentido, y siguiendo a Chomsky (1995, 136), la concordancia estaría por encima del verbo: [AgrP [Agr [VP [Adv [VP [V]]]]]]. En la propuesta de Preminger (2014), basada en el modelo de obligatory operations, la búsqueda de un rasgo particular es una relativized probing.

17 En inglés, por ejemplo, aunque el número y la persona contribuyen a la interpretación en la FD, no contribuyen en el V (Pesetsky y Torrego 2007, 263-264). 
(Adv-V). Como indica van Gelderen $(2008,187)$, ello estaría en relación con el Principio de Economía, ya que para el sistema computacional es más sencillo trabajar con rasgos gramaticales que con rasgos semánticos. ${ }^{18}$

En nuestro caso, el adverbio siempre puede concordar (+ - $n)$ con el verbo (V-n) porque el verbo es el elemento que está más cerca (closeness). En estos casos el verbo finito parece ser un controlador de concordancia, aunque es sabido que técnicamente el sujeto es el elemento que controla la concordancia. Con todo, la - $n$ de siempren sería un marcador de concordancia, pero entendida como una instancia de múltiple agreement. De hecho, este análisis formal podría dar cuenta de por qué muchos adverbios temporales (y locativos) pueden manifestar concordancia plural, como luegon (luego-n, cf. 11) y todavían (todavía-n, cf. 12) en algunas variedades vernaculares, pero parece que otros no, como tempranon (temprano- $n$ ), tarden (tarde- $n$ ) o despuesen (después-n), ya que estos últimos suelen aparecer pospuestos al verbo con mayor frecuencia que los primeros, y ya vimos que la posición preverbal favorece la concordancia plural (-n).

(11) a. A partir de las 7 am dan números, hasta 400. Luegon te citan a una cierta hora para tomarte los datos y a las 2 hs te lo entregan (Argentina).

b. con unos kilitos de más, pero con un aspecto muy saludable. Para que luegon digan que hay que estar super delgada para ser bella. Fíjense en Demi Lovato (República Dominicana).

c. La historia de amor entre estos dos acabó perfectamente y luegon tuvieron una preciosa amistad, deberían haberlo dejado alli (España).

(12) a. este año fue el año por exelencia [sic] de visitas internacionales, y todavían quedan muchas mas por llegar, con los precios por las nubes pero aun asi (México).

b. De las radios que figuran solo quedan Panamericana y Miraflores y todavían transmiten en AM en simultaneo (Perú).

c. Campanella apostó a la vieja escuela y demostró que los métodos arcaicos todavían rinden frutos. En este caso grabó las voces con todos los actores juntos en una sala (Argentina).

En cuarto lugar, y por último, desde el punto de vista de la producción del lenguaje, el orden en que se lleva a cabo la operación de inserción del elemento (- $n$ ) es interesante para comprender mejor los procesos de secuenciación y selección de elementos, así como los procesos de ordenamiento durante la planificación del habla (Garret 1980, Igoa 1996).

En concreto, Igoa, García-Albea y Sánchez-Casas (1999, 174-176) recuerdan que los procesos de asignación de género ocurren en el léxico, mientras que la asignación de número no. De hecho, los rasgos de número se asignan de manera independiente a los lemas y después a las palabras, a través de una operación de construcción de estructura de frase. Por ello, y dada

18 En varios trabajos se ha indicado que el circunstancial se adjunta como el complemento más interno de los verbos (cf. Kayne 1994; Larson 2004). En atkan aleut (lengua esquimal de Alaska), por ejemplo, los adverbios circunstanciales pueden coincidir en las características phi con una FD más allá del verbo principal, es decir, el adverbio puede concordar con el sujeto: Hlas (chico.3.pl.) waaGalakan (volver.Neg-Conj.) qilaXsis (mañana.3.pl.), 'Los chicos no volvieron esta mañanas' (Nilsen 2000, 104). 
la dependencia del número gramatical del número nocional, es plausible afirmar que las inflexiones numéricas no se recuperan necesariamente con representaciones de lemas, sino por otros medios. Para estos autores, por tanto, las características numéricas se especifican en un nivel conceptual preverbal y se asignan como una característica abstracta al árbol de estructura de frase durante la codificación de funciones gramaticales.

Por otro lado, tal y como ha indicado del Viso (2002, 378-379), mientras que el género estaría más íntimamente ligado a la raíz, por lo que tiende a moverse junto con ella, el número tiende a aparecer en su lugar correcto, es decir "más independiente del movimiento que pueda sufrir la raíz a la que debía adjuntarse". De hecho, en los errores de desplazamiento (como el de siempren) los sufijos que se desplazan son el número y algunos sufijos verbales, y afectan sobre todo a palabras de clase cerrada (en un $91 \%$ de los casos) ${ }^{19}$.

\section{Conclusiones}

La realización de este trabajo permite obtener una serie de conclusiones que a continuación mencionamos.

El trabajo ha mostrado que la pluralización del adverbio (-n) es un fenómeno posible en español actual. Las lenguas y los dialectos que muestran concordancia adverbial, como es el caso de algunas variedades del español (siempren), del gallego y del catalán (sempren), estarían en una etapa sintáctica más "avanzada", según la jerarquía de implicación de Moravcsik (1974), pues la concordancia adverbial es la última de las posibilidades.

Siguiendo los trabajos de psicolingüística, la 'pluralidad' (o número gramatical) se deriva - en la mayoría de los casos- de un rasgo semántico de número nocional o [numerosidad] y se representa como parte de las características gramaticales de una palabra, reflejando así las relaciones de concordancia (Igoa, García-Albea y Sánchez-Casas 1999), ya sea entre un elemento controlador y su target (Corbett 2006), ya sea porque dos palabras están relacionadas con una entidad cognitiva que porta ese rasgo (Kibrik 2019). En cualquier caso, es evidente que el orden de palabras juega un papel crucial en el procesamiento de la información adverbio-verbo. En el caso concreto que nos ocupa, la posición con respecto al verbo resulta ser categóricamente preverbal en siempren $(93,02 \%)$, frente a la posición postverbal $(3,10 \%)$. Del mismo modo, la forma siempre también muestra una preferencia alta por la posición preverbal en español actual: 67,55\% (629.879 casos), frente a un 32,45\% (302 620 casos) de la postverbal, según los datos del Corpus del español: Web/Dialectos.

Por último, soy consciente de que quedan varios aspectos (algunos socioculturales, y otros quizá de contacto de lenguas, como para el caso de Perú

19 Además, "siempre que un error, de la clase que sea, afecta a dos palabras de distinto género y/o número gramatical, el género y/o número de las palabras de las categorías menores será el apropiado para la palabra con la cual deben establecer concordancia, aunque ésta esté ubicada erróneamente" (del Viso 2002, 379). 
y otros países) por aclarar sobre la pluralización (-n) de los adverbios en español, así como el estudio de otras formas que no han sido tratadas en esta ocasión. En un futuro trabajo se podrá dar cuenta, por tanto, de otros casos de pluralización adverbial, como nuncan (Espero que nuncan olviden esas palabras) o enciman (El problema es que permiten todo y enciman se ríen), entre otros, y ofrecer una explicación interdisciplinar que permita comprender estos casos de información redundante (Adv-n $+V-n)$. 


\section{Q Bibliografía}

" Ackema, Peter y Ad Neeleman. 2004. Beyond morphology. Interface conditions on word formation. Nueva York: Oxford University Press.

》Asociación de Academias de la Lengua Española. 2010. Diccionario de americanismos. Lima: Santillana.

» Bock, Kathryn et al. 2001. "Some attractions of verb agreement". Cognitive Psychology 43/2: 83-128.

"Chomsky, Noam. 1995. The Minimalist Program. Cambridge, MA: MIT Press.

"Cinque, Guglielmo. 1999. Adverbs and functional heads: A cross-linguistic perspective. Oxford: Oxford University Press.

》Corbett, Greville G. 2006. Agreement. Cambridge: Cambridge University Press.

» Davies, Mark. 2016. Corpus del español. Provo, UT: Brigham Young University. https:// www.corpusdelespanol.org

" del Viso, Susana. 2002. "Los lapsus linguae como fuente de datos en el estudio de la producción del lenguaje: un corpus de errores en castellano". Anuario de Psicología 33/3: 355-384.

"Felíu Arquiola, Elena y Enrique Pato. 2019. “¿Realmentes existen?: la "pluralización” de los adverbios en -mente en español actual”. Onomázein 44: 166-190.

» Felíu Arquiola, Elena y Enrique Pato. 2020. En torno a la "concordancia adverbial" en español: tres casos de variación. Madrid: CSIC.

» Fontanella de Weinberg, M. Beatriz. 1993. El español de América. Madrid: Editorial Mapfre.

" Garret, Merrill F. 1980. "Levels of processing in sentence production". En Language production: Speech and Talk, editado por Brian Butterworth, 177-220. Nueva York/Londres: Academic Press.

" Hernández, Humberto. 2004. El mensaje en los medios. A propósito del estudio lingüístico de la prensa regional canaria. Madrid/Frankfurt: Iberoamericana/Vervuert.

" Igoa, José M. 1996. “The relationship between conceptualization and formulation processes in sentence production: some evidence from Spanish". En Language processing in Spanish, editado por Manuel Carreiras, José E. García-Albea y Nuria Sebastián, 305351. Mahwah, NJ: Erlbaum.

"Igoa, José M., J. Eugenio García-Albea y Rosa Sánchez-Casas. 1999. “Gender-number dissociations in sentence production in Spanish". Rivista di Linguistica 11/1: 163-196.

»Kany, Charles E. 1969.Sintaxis hispanoamericana. Madrid: Gredos.

" Kayne, Richard S. 1994. The antisymmetry of syntax. Cambridge, MA: MIT Press.

" Kibrik, Andrej A. 2019. "Rethinking agreement: Cognition-to-form mapping”. Cognitive Linguistics 30/1: 37-83.

" Larson, Richard K. 2004. “Sentence-final adverbs and 'scope'”. En NELS 34. Proceedings of the 34th annual meeting of the North East Linguistic Society, editado por Keir Moulton y Matthew Wolf, 23-43. University of Massachusetts: GLSA. 
" Lecours, André R. y François Lhermitte. 1969. "Phonemic paraphasia: linguistic structures and tentative hypotheses". Cortex 5/2: 193-228.

» Lipski, John M. 1996. El español de América. Madrid: Cátedra.

" Mare, María. 2018. “Una nueva mirada sobre la concordancia inesperada en español”. Revista de Filología Española XCVIII: 397-422.

" Moravcsik, Edith A. 1974. "Object-verb agreement". Working papers on language universals 15: 25-140.

"Nilsen, Øystein. 2000. The syntax of circumstantial adverbials. Oslo: Novus.

"Pato, Enrique (dir.). 2014-2020. Corpus oral de la lengua española en Montreal(COLEM). Montreal: Université de Montréal. https://esp-montreal.jimdo.com

》Pato, Enrique. 2019. "Cadas cosas se leen y cadas fantasma hay en la red: La "pluralización' del cuantificador cada en español actual”. Borealis 8/2: 225-242.

"Pato, Enrique y Vanessa Casanova. 2017. "El hotel está muy cercas de la playa, o la falsa pluralización del adverbio cerca en el español de México”. Anuario de Letras. Lingüística y Filología V/1: 149-169.

"Pesetsky, David y Esther Torrego. 2007. "The syntax of valuation and interpretability of features". En Phrasal and clausal architecture, editado por Simin Karimi, Vida Samiian y Wendy Wilkins, 262-294. Amsterdam/Philadelphia: John Benjamins.

"Preminger, Omer. 2014. Agreement and lts Failures. Cambridge, MA: MIT Press.

》Real Academia Española. Banco de datos (CORDE). Corpus diacrónico del español. Madrid: RAE. http://www.rae.es

" Real Academia Española. Corpus del español del siglo XXI (CORPES XXI), [versión o.9]. Madrid: RAE. http://www.rae.es

"Real Academia Española y Asociación de Academias de la Lengua Española. 2005. Diccionario panhispánico de dudas. Madrid: Santillana.

»Real Academia Española y Asociación de Academias de la Lengua Española. 2009. Nueva gramática de la lengua española. Madrid: Espasa.

"Real Academia Española y Asociación de Academias de la Lengua Española. 2017. Diccionario de la lengua española. Edición del Tricentenario. Madrid: RAE. http://www.rae.es

"Van Gelderen, Elly. 2008. "Economy of Merge and grammaticalization: Two steps in the evolution of language". En Variation, selection, development: probing the evolutionary model of language change, editado por Regine Eckardt, Gerhard Jäger y Tonjes Veenstra, 179-197. Berlín: Walter de Gruyter. 